Article

\title{
Stochastic Bifurcation of a Strongly Non-Linear Vibro-Impact System with Coulomb Friction under Real Noise
}

\author{
Li Liu ${ }^{1}$, Wei $\mathrm{Xu}^{1, *}$, Xiaole Yue ${ }^{1}$ and Dongmei Huang ${ }^{2}$ \\ 1 Department of Applied Mathematics, Northwestern Polytechnic University, Xi'an 710072, China; \\ liuli0951@126.com (L.L.); xiaoleyue@nwpu.edu.cn (X.Y.) \\ 2 School of Mathematics and Statistics, Xidian University, Xi'an 710071, China; dongmeihuang1@hotmail.com \\ * Correspondence: weixu@nwpu.edu.cn; Tel.: +86-2988492393
}

Received: 18 November 2018; Accepted: 20 December 2018; Published: 21 December 2018

\begin{abstract}
This manuscript investigated the response of a strongly non-linear vibro-impact (VI) system with Coulomb friction. The impact model is used with classical impact. The excitation is modelled by real noise. First, the VI system is converted into a simplified system without any barrier by non-smooth transformation (symmetric transformation). The stochastic averaging method is adopted to obtain the theoretical stationary probability function of the VI system. Next, the Duffing Van der Pol VI system with Coulomb friction is used to verify the validity of the proposed theoretical method compared with numerical simulations. Moreover, the influence of bandwidth, noise intensity, and friction amplitude are further analyzed in detail on the probability density function (PDF) of distribution of the VI system. The P-bifurcation is studied by a qualitative change of friction amplitude and restitution coefficient on the stationary probability distribution, which indicated that these parameters can arouse the emergence of stochastic P-bifurcation.
\end{abstract}

Keywords: vibro-impact system; Coulomb friction; stochastic response; stochastic P-bifurcation

\section{Introduction}

\subsection{Background}

In the field of science and engineering, vibro-impact (VI) systems can model some relevant problems such as ship roll motion against icebergs, rotor-stator rubbing in rotating machinery, or impact interaction in pipe-baffle interfaces due to seismic excitations and so on [1]. Moreover, the VI systems displayed some interesting phenomena, such as grazing bifurcation [2], torus bifurcation [3], and chatter and sticking [4]. Some modelling techniques are developed to model impact, such as the Herz contact law, for classical impact with an instantaneous velocity jump. Because excessive impacts may cause damage to some structural components in practice, in order to avoid harmful impact or some random vibro-impact, research on the response and bifurcation of vibro-impact has received much attention.

\subsection{Literature Survey}

Namachachivaya [5,6] studied the dynamic behaviors of VI systems under random perturbation. Huang [7] investigated the stationary response for stochastically excited multi-degree-of-freedom VI systems. Xu [8] studied the stochastic response of VI systems with inelastic contact. Rong [9] applied multiple scales to study the amplitude-frequency response of VI systems. Yang [10] investigated the random response of Rayleigh VI systems excited by Poisson white noise. Zhu [11,12] studied the random response of VI systems through the exponential-polynomial closure method. 
As another representative non-smooth factor, friction, which exists in the mechanical system, has been extensively researched in the last two decades. For example, Green [13] analyzed the effect of friction on the response of an electromagnetic energy harvester with Duffing-type non-linearities. Sun [14] applied generalized cell mapping to investigate the random response of the Coulomb friction system. Pankaj [15] studied the stochastic bifurcation of a Duffing oscillator with Coulomb friction under Poisson white noise. Sun [16] researched the reliability of a non-linearly damped oscillator with Coulomb friction under Gaussian white noise excitation. If the system response is required to be below a critical level for safety consideration, a control device may be needed. Rigatos $[17,18]$ studied the sensorless control and Bryson [19] considered the optimal control; Pappalardo [20,21] investigated the algorithm controlling the mechanical system.

\subsection{Formulation of the Problem}

In addition, in some mechanical systems, such as rotor-stator interactions in turbines, interacting gear teeth, riveted or bolted supports in structural systems [22,23], vibro-impact, and friction dynamics arise simultaneously when some rigid subparts of mechanical systems move separately in partial-motion restraining mechanisms. The friction and impact between gear wheels and railways usually induces the "chatter" phenomenon, which tends to generate undesired noise and excessive vibrations which could influence performance and even cause damage to the system.

Since random factors in most real-world circumstances may influence the dynamic behavior of VI systems, some researchers studied VI systems under random excitation. For mathematical simplicity, the random driving force is generally assumed as zero mean Gaussian white noise. However, white noise has an idealization of the correlation time scale, while practical physical models need to consider finite correlation times, so the white noise does not describe random disturbances in nature. Wide-band noise as a finite correlation of time stochastic excitation has received increasing attention. Zhu [24] studied the dynamic behaviors of strongly non-linear systems under broad noise by averaging method; Lin and Zhu $[25,26]$ researched the response and stability for wide-band noise for an excited viscoelastic system.

\subsection{Contribution of this Study}

As mentioned above, the random vibration of VI systems has attracted much attention in the past. However, the investigation hasn't considered friction in the VI system. Moreover, the existence of friction in the VI system is unavoidable, and the random excitation modeled by real noise is more reasonable. Bifurcation analysis under different system parameters is essential to avoid the appearance of undesirable dynamic behaviors. Thus, it is significant to investigate the stochastic response and bifurcation of the VI system with Coulomb friction subjected to real noise excitation.

\subsection{Organization}

In this paper, the stochastic response and bifurcation of a strongly non-linear VI system with Coulomb friction under real noise excitation is investigated. The model of classical impact with instantaneous velocity jump is used in this paper. In the first step, the VI system is converted into the simplified system without any barrier by non-smooth transformation. In the second step, the stationary probability density function (PDF) is obtained through the stochastic averaging method. The effects of bandwidth, noise intensity of wideband noise, and friction parameters on stationary probability density are considered. Lastly, stochastic P-bifurcation is studied by the variation of friction parameters and restitution coefficient on the stationary probability distribution. 


\section{System Description and Non-Smooth Transformation}

\subsection{System Description}

Consider the strongly non-linear VI system with Coulomb Friction (Figure 1) subjected to real noise excitation

$$
\left\{\begin{array}{l}
\ddot{x}+\varepsilon c(x, \dot{x}) \dot{x}+g(x)+\mu \operatorname{sgn}(\dot{x})=\varepsilon^{1 / 2} f_{i}(x, \dot{x}) \xi_{i}(t) \quad x>b, \\
\dot{x}_{+}=-r \dot{x}_{-} \quad x=b .
\end{array}\right.
$$

where $\ddot{x}, \dot{x}$ and $x$ denote the acceleration, velocity, and displacement. Here $c(x, \dot{x})$ is the damping coefficient, $g(x)$ is the strongly non-linear stiffness and is an odd function. $b$ denotes distance of the unilateral barrier from the static equilibrium position of the system and we assigned zero to $b$ in this paper. $\dot{x}_{+}$and $\dot{x}_{-}$denote impact velocities before and after impact. $\varepsilon$ is a small scale parameter, and $r(0<r \leq 1)$ is the restitution coefficient. $\xi_{i}(t)$ represents real noise with correlation function $R_{i j}(\tau)$. Coulomb friction $\mu \operatorname{sgn}(\dot{x})$ has constant amplitude $\mu$ and $\operatorname{sgn}(\cdot)$ denotes sign function

$$
\operatorname{sgn}(\dot{x})=\left\{\begin{array}{ll}
1 & \dot{x}>0 \\
0 & \dot{x}=0 \\
-1 & \dot{x}<0
\end{array} .\right.
$$

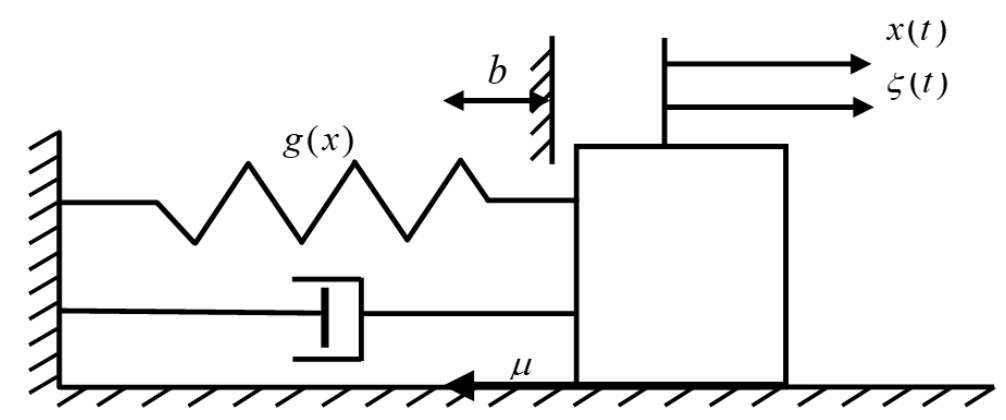

Figure 1. Vibro-impact system with Coulomb friction.

Although Coulomb friction is intuitively simple, its discontinuity added difficulty in trying to analytically evaluate the system response.

\subsection{Non-Smooth Transformation}

The procedure of non-smooth transformations mentioned in Ref. [27] are introduced as follows

$$
x=|y|=x_{1}, \quad \dot{x}=\dot{y} \operatorname{sgn}(y)=x_{2}
$$

The transformation of Equation (3) maps the domain $x>0$ of original phase plane $(x, \dot{x})$ onto the whole phase plane $(y, \dot{y})$; the transformed equations of new variables can be written as:

$$
\left\{\begin{array}{l}
\ddot{y}+\varepsilon c(y, \dot{y}) \dot{y}+g(y)+\mu \operatorname{sgn}(\dot{x})=\varepsilon^{\frac{1}{2}} f_{i}(y, \dot{y}) \xi_{i}(t) \operatorname{sgn}(y) \quad y\left(t_{\bullet}\right) \neq 0 \\
\dot{y}_{+}=r \dot{y}_{-} \quad y\left(t_{\bullet}\right)=0
\end{array}\right.
$$

Through the new equation, one can find that the jump of transformed velocity $\dot{y}$ changes proportional to $(1-r)$ instead of $(1+r)$ for the original velocity $\dot{x}$. By virtue of $y\left(t_{*}\right)=0$ and $\delta\left(t-t_{*}\right)=|\dot{y}(t)| \delta(y(t))$ at impact, Equation (2) can be considered as an additional impulse through Dirac delta function and the impulsive term can be written as

$$
\left(\dot{y}_{-}-\dot{y}_{+}\right) \delta\left(t-t_{*}\right)=(1-r) \dot{y}|\dot{y}| \delta(y)
$$


The term $(1-r) \dot{y}|\dot{y}| \delta(y)$ tends to depict the impact losses of the system. Thus, Equation (1) and (2) can be combined into one equation by adding the impulsive damping term $(1-r) \dot{y}|\dot{y}| \delta(y)$ through the transformation (5)

$$
\begin{aligned}
& \ddot{y}+\varepsilon(f(y, \dot{y})) \dot{y}+\mu \operatorname{sgn}(\dot{y})+g(y)=\varepsilon^{\frac{1}{2}} f_{i}(y, \dot{y}) \xi_{i}(t) \operatorname{sgn}(y), \\
& f(y, \dot{y})=c(y, \dot{y})+\frac{1}{\varepsilon}(1-r)|\dot{y}| \delta(y), \quad U(y)=\int_{0}^{y} g(u) d u .
\end{aligned}
$$

\section{Stochastic Averaging Procedure}

We can assume that the solution of Equation (6) has the following form [25]:

$$
\left\{\begin{array}{l}
y(t)=A(t) \cos \theta(t) \\
\dot{y}(t)=-A(t) v(A, \theta) \sin \theta(t) \\
\theta(t)=\phi(t)+\varphi(t)
\end{array}\right.
$$

where $A, \theta, \phi, \varphi$ are all random processes, $v(A, \theta)$ is the instantaneous frequency of the oscillator and has the following form:

$$
v(A, \theta)=\frac{d \phi}{d t}=\sqrt{\frac{2(U(A)-U(A \cos \theta))}{A^{2} \sin ^{2} \theta}}=b_{0}(a)+\sum_{r=1}^{\infty} b_{r}(A) \cos r \theta,
$$

Substituting Equation (7) into Equation (6), we can observe the transformation equations of amplitude $A(t)$ and phase angle $\varphi(t)$ :

$$
\begin{gathered}
\frac{d A}{d t}=\varepsilon F_{1}+\varepsilon^{1 / 2} G_{1 k}(A, \varphi) \xi_{k}(t), \frac{d \varphi}{d t}=\varepsilon F_{2}+\varepsilon^{1 / 2} G_{2 k}(A, \varphi) \xi_{k}(t), \\
F_{1}=\frac{-A^{2} 2^{2} \sin ^{2} \theta}{g(A)} f(A \cos \theta,-A v \sin \theta)-\frac{A v \sin \theta}{g(A)} \frac{\mu}{\varepsilon} \operatorname{sgn}(-A v \sin \theta), \\
F_{2}=\frac{-A v^{2} \sin \theta \cos \theta}{g(A)} f(A \cos \theta,-A v \sin \theta)-\frac{v \cos \theta}{g(A)} \frac{\mu}{\varepsilon} \operatorname{sgn}(-A v \sin \theta), \\
G_{1 k}=-\frac{A v \sin \theta}{g(A)} f_{k}(A \cos \theta,-A v \sin \theta), G_{2 k}=-\frac{v \cos \theta}{g(A)} f_{k}(A \cos \theta,-A v \sin \theta) .
\end{gathered}
$$

According to the Stratonovich-Khasminskii limit theorem [28,29], the process $A(t)$ can be approximated as a Markov process and is governed by the following averaged Itô stochastic differential equation:

$$
d A=m(A) d t+\sigma(A) d B(t)
$$

where

$$
\begin{gathered}
m(A)=\varepsilon\left\langle F_{1}+\int_{-\infty}^{0}\left[\left.\left.\frac{\partial G_{1 k}}{\partial A}\right|_{t} G_{1 j}\right|_{t+\tau}+\left.\left.\frac{\partial G_{1 k}}{\partial \theta}\right|_{t} G_{2 j}\right|_{t+\tau}\right] R_{k j}(\tau) d \tau\right\rangle_{t} \\
\sigma^{2}(A)=\varepsilon\left\langle\left.\left.\int_{-\infty}^{+\infty} G_{1 k}\right|_{t} G_{1 j}\right|_{t+\tau} R_{k j}(\tau) d \tau\right\rangle_{t}
\end{gathered}
$$

$B(t)$ denotes a unit wiener process, \langle\rangle$_{t}$ represents the time averaging.

To obtain the explicit expressions of the averaged $m(A)$ an $\sigma^{2}(A)$ in Equation (10) and (11), one can expand $\varepsilon F_{1}$ and $\varepsilon^{1 / 2} G_{i k}$ into Fourier series with respect to $\theta$ :

$$
\left\{\begin{array}{l}
\varepsilon F_{1}(A, \Gamma)=F_{10}+\sum_{i=1}^{\infty}\left(F_{1 i}^{(s)} \cos i \theta+F_{1 i}^{(t)} \sin i \theta\right) \\
\varepsilon^{1 / 2} G_{j k}(A, \Gamma)=G_{j k 0}+\sum_{i=1}^{\infty}\left(G_{j k i}^{(s)} \cos i \theta+F_{j k i}^{(t)} \sin i \theta\right) \\
j=1,2 ; \quad k=1,2, \cdots, m
\end{array}\right.
$$

Using Equation (12) and time averaging, we obtained the drift and diffusion coefficients given by: 


$$
\begin{gathered}
m(A)=F_{10}(A)+\frac{\pi}{2} \sum_{i=1}^{\infty}\left\{\left[\frac{d G_{1 k i}^{(s)}}{d A} G_{1 l i}^{(s)}+\frac{d G_{1 k i}^{(t)}}{d A} G_{1 l i}^{(t)}+i\left(G_{1 k i}^{(t)} G_{2 l i}^{(s)}-G_{1 k i}^{(s)} G_{2 l i}^{(t)}\right)\right] S_{k l}(i \omega(A))\right. \\
\left.+\left[\frac{d G_{1 k i}^{(s)}}{d A} G_{1 l i}^{(t)}-\frac{d G_{1 k i}^{(t)}}{d A} G_{1 l i}^{(s)}+i\left(G_{1 k i}^{(t)} G_{2 l i}^{(t)}+G_{1 k i}^{(s)} G_{2 l i}^{(s)}\right)\right] I_{k l}(i \omega(A))\right\}+\pi \frac{d G_{1 k 0}}{d A} G_{1 l 0} S_{k l}(0) \\
\sigma^{2}(A)=2 \pi G_{1 k 0} G_{1 l 0} S_{k l}(0)+\pi \sum_{i=1}^{\infty}\left[\left(G_{1 k i}^{(s)} G_{1 l i}^{(s)}+G_{1 k i}^{(t)} G_{1 l i}^{(t)}\right) S_{k l}(i \omega(A))+\right. \\
\left.\left(G_{1 k i}^{(s)} G_{1 l i}^{(t)}-G_{1 k i}^{(t)} G_{1 l i}^{(s)}\right) I_{k l}(i \omega(A))\right]
\end{gathered}
$$

where

$$
S_{k l}(\omega)=\frac{1}{\pi} \int_{-\infty}^{0} R_{k l}(\tau) \cos \omega \tau d \tau \quad I_{k l}(\omega)=\frac{1}{\pi} \int_{-\infty}^{0} R_{k l}(\tau) \sin \omega \tau d \tau
$$

\section{Response Probability Density Functions}

The PDF for amplitude is governed by the following Fokker-Planck-Kolmogorov (FPK) equation:

$$
\frac{\partial p}{\partial t}=-\frac{\partial}{\partial A}[m(A) p]+\frac{1}{2} \frac{\partial^{2}}{\partial A}\left[\sigma^{2}(A) p\right]
$$

where the boundary condition is:

$$
\begin{gathered}
p=\text { finite as } A=0, \\
p \rightarrow 0, \partial p / \partial A \rightarrow 0 \text { as } A \rightarrow \infty .
\end{gathered}
$$

According to Zhu [24], $N$ is a normalization constant and the stationary solution of FPK equation is

$$
p(A)=\frac{N}{\sigma^{2}(A)} \exp \left[\int_{0}^{A} \frac{2 m(u)}{\sigma^{2}(u)} d u\right],
$$

The stationary probability of $E$ can be derived from the Equation (14) as follows:

$$
p(E)=p(A)\left|\frac{d A}{d E}\right|=\left.\frac{p(A)}{g(A)}\right|_{A=U^{-1}(E)},
$$

where $A=U^{-1}(E)$ is the inverse function of $U(A)=E$, the stationary probability density of velocity and displacement can be further obtained from $p(E)$ as follows:

$$
p(y, \dot{y})=\left.\frac{p(E)}{T(E)}\right|_{E=(1 / 2) \dot{x}^{2}+U(x)},
$$

where

$$
T(E)=\left.\frac{2 \pi}{b_{0}(A)}\right|_{A=U^{-1}(E)} .
$$

Through the transformation (3), the joint stationary PDF of the original displacement $x_{1}$ and velocity $x_{2}$ can be expressed as

$$
p\left(x_{1}, x_{2}\right)=2 \widetilde{p}_{y_{1}, y_{2}}\left(x_{1}, x_{2}\right)
$$

The stationary PDFs of displacement $x_{1}$ and velocity $x_{2}$ are derived, respectively:

$$
p_{x_{1}}\left(x_{1}\right)=\int_{-\infty}^{+\infty} p\left(x_{1}, s\right) d s, \quad p_{x_{2}}\left(x_{2}\right)=\int_{-\infty}^{+\infty} p\left(x_{2}, s\right) d s .
$$




\section{Example}

The Duffing-Van der Pol VI system with Coulomb Friction are often met in science and engineering as a representative model of a strongly non-linear system under real noise excitation, which has the following form:

$$
\left\{\begin{array}{l}
\ddot{x}+\left(c_{2} x^{2}-c_{1}\right) \dot{x}+\omega_{0}^{2} x+\alpha x^{3}+\mu \operatorname{sgn}(\dot{x})=f \xi(t) \quad x>0, \\
\dot{x}_{+}=r \dot{x}_{-} \quad x=0
\end{array}\right.
$$

where $c_{1}, c_{2}, \alpha, f$ and $\omega_{0}$ are positive parameters, $\xi(t)$ is the real noise and the spectral density has the following low-pass type

$$
S(\omega)=\frac{D}{\pi} \frac{1}{\omega^{2}+\alpha_{1}^{2}}
$$

where $\alpha_{1}$ and $D$ are the bandwidth and noise intensity of the real noise. With the above spectral density the random excitation $\xi(t)$ can be generated from a first filter

$$
\dot{\xi}(t)+\alpha \xi(t)=W(t)
$$

where $W(t)$ is a white noise with noise intensity $2 D$. According to Equation (6), the equation of system (17) can be transformed as the following stochastic system

$$
\ddot{y}+\left(c_{2} y^{2}-c_{1}+(1-r)|\dot{y}| \delta(y)\right) \dot{y}+\mu \operatorname{sgn}(\dot{y})+\omega_{0}^{2} y+\alpha y^{3}=f \xi(t) \operatorname{sgn}(y),
$$

The instantaneous frequency $v(A, \theta)$ in Equation (8) can be approximated as the following finite sum.

$$
\begin{gathered}
v(A, \theta)=b_{0}(A)+b_{2}(A) \cos 2 \theta+b_{4}(A) \cos 4 \theta+b_{6}(A) \cos 6 \theta \\
b_{0}(A)=\sqrt{\left(\omega_{0}^{2}+\frac{3}{4} \alpha A^{2}\right)}\left(1-\frac{\eta^{2}}{16}\right), \quad b_{2}(A)=\sqrt{\left(\omega_{0}^{2}+\frac{3}{4} \alpha A^{2}\right)}\left(\frac{\eta}{2}+\frac{3 \eta^{3}}{64}\right), \\
b_{4}(A)=\sqrt{\left(\omega_{0}^{2}+\frac{3}{4} \alpha A^{2}\right)}\left(-\frac{\eta^{2}}{16}\right), \quad b_{6}(A)=\sqrt{\left(\omega_{0}^{2}+\frac{3}{4} \alpha A^{2}\right)}\left(\frac{3 \eta^{3}}{64}\right) .
\end{gathered}
$$

where

$$
\eta=\left(\alpha a^{2} / 4\right) /\left(\omega_{1}^{2}+3 \alpha a^{2} / 4\right) .
$$

Equation (18) indicates the average frequency is $\omega(a)=b_{0}(a)$. Thus, applying the stochastic averaging method, we obtained drift and diffusion coefficients of Equation (9) defined as

$$
\begin{gathered}
\left.m(A)=\frac{A^{2}}{8 g(A)}\left[\left[\varepsilon c_{1}\left(4 \omega_{0}^{2}+5 \alpha A^{2} / 2\right)-\varepsilon c_{2} A^{2}\left(\omega_{0}^{2}+3 \alpha A^{2} / 4\right)\right]\right]\right] \\
-\frac{A^{2}}{8 g(A)}\left[8 \pi(1-r)\left(\omega_{0}^{2}+\alpha A^{2} / 2\right)\left(b_{0}(A)-b_{2}(A)+b_{4}(A)-b_{6}(A)\right]\right. \\
-\frac{A \mu}{\pi g(A)}\left[2 b_{0}(A)-\frac{2}{3} b_{2}(A)-\frac{2}{15} b_{4}(A)-\frac{2}{35} b_{6}(A)\right]+\frac{\pi A}{8 g(A)} \times \\
\left\{\left(4 b_{0} d_{0}-2 b_{2} d_{0}-2 b_{0} d_{2}+b_{2} d_{2}\right) S(\omega(A))+\left(b_{2} d_{2}-b_{4} d_{2}-b_{2} d_{4}+b_{4} d_{4}\right) S(3 \omega(A))\right. \\
\left.+\left(b_{4} d_{4}-b_{6} d_{4}-b_{4} d_{6}+b_{6} d_{6}\right) S(5 \omega(A))+b_{6}(A) S_{1}(7 \omega(A)) d_{6}\right\}+\frac{\pi A}{8 g^{2}(A)} \times \\
{\left[\left(4 b_{0}^{2}-b_{2}^{2}\right) S(\omega(A))+3\left(b_{2}^{2}-b_{4}^{2}\right) S(3 \omega(A))+5\left(b_{4}^{2}-b_{6}^{2}\right) S(\omega 5(A))+7 b_{6}^{2} S(7 \omega(A))\right],} \\
\sigma^{2}(A)=\frac{\pi A^{2}}{4 g^{2}(A)}\left\{\left(2 b_{0}-b_{2}\right)^{2} S(\omega(A))+\left(b_{2}-b_{4}\right)^{2} S(3 \omega(A))\right. \\
+\left(b_{4}-b_{6}\right)^{2} S(5 \omega(A))+b_{6}^{2} S(7 \omega(A)) .
\end{gathered}
$$

where

$$
d_{i}=\frac{d}{d A}\left[b_{i}(A) \frac{A}{g(A)}\right], \quad(i=0,2,4,6) .
$$

\subsection{The Effect of System Parameters on Response}

In order to verify the accuracy of the proposed analytical method, the analytical stationary probability density was compared with the numerical results based on the original system (17). 
When system parameters were chosen as $c_{2}=0.05, c_{1}=0.025, \alpha=0.6, r=0.96, \omega_{0}=1.0$, the effect of the magnitude parameter $\alpha_{1}$, friction amplitude $\mu$, and noise intensities $D$ on the responses of the system (17) were analyzed, respectively.

When $\mu=0.001, \alpha_{1}=2.0$ is fixed, all stationary PDFs of amplitude $A$, displacement $x_{1}$, and velocity $x_{2}$ for different noise intensities $D$ are shown respectively in sub-captions (a-c) of Figure 2 . These figures indicated that the influence of noise intensity on the response of system (17) is existent. Concretely, the increase of noise intensity $D$ leads to the reduction of all stationary PDFs of amplitude, displacement, and velocity.

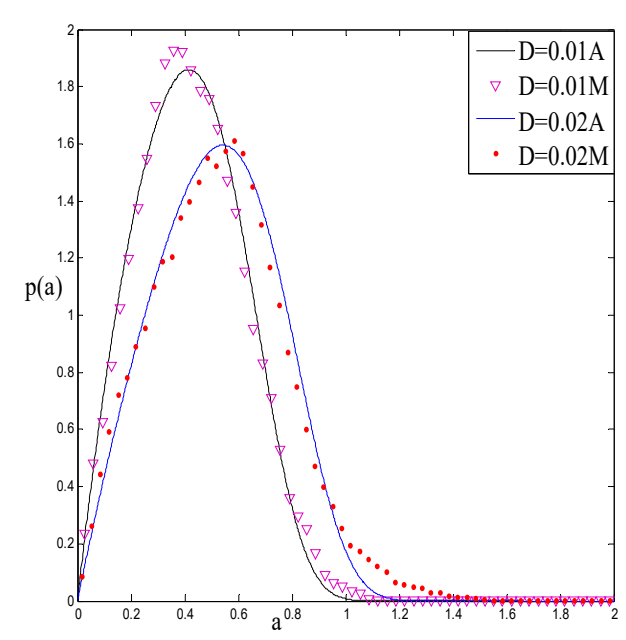

(a)

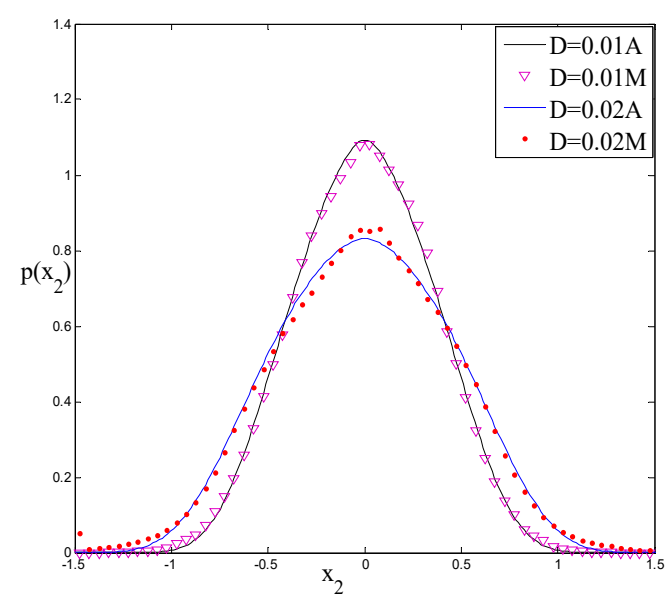

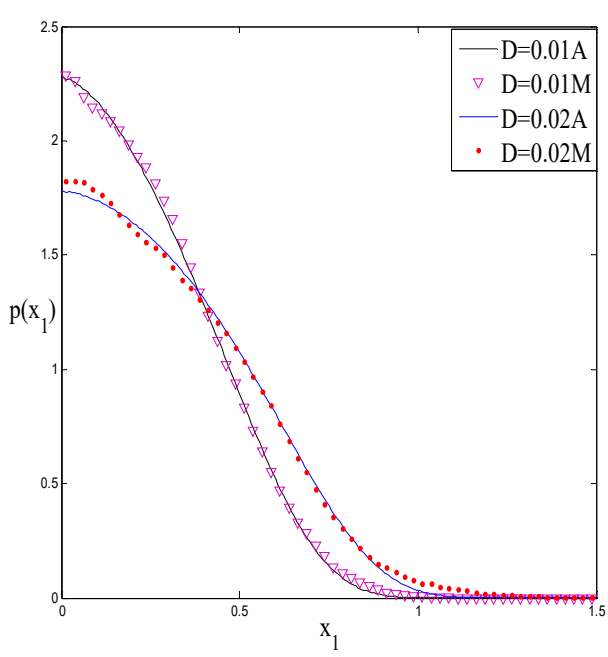

(b)

(c)

Figure 2. Numerical and analytical stationary probability density function (PDF) for $\alpha_{1}=2.0, \mu=0.001$. (a) PDF of amplitude A. (b) PDF of displacement $x_{1}$. (c) PDF of velocity $x_{2}$.

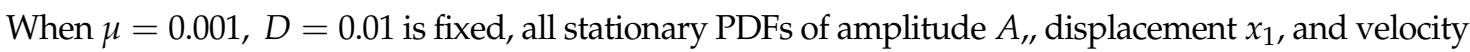
$x_{2}$ for different bandwidths $\alpha_{1}$ are shown respectively in sub-captions (a-c) of Figure 3. A larger bandwidth $\alpha_{1}$ corresponds to a higher peak of all stationary PDFs in Figure 3. When $D=0.01, \quad \alpha_{1}=2.0$ is fixed, the stationary PDFs of amplitude $A$, displacement $x_{1}$, and velocity $x_{2}$ for different friction parameters are shown respectively in sub-captions $(\mathrm{a}-\mathrm{c})$ of Figure 4 . Concretely, the increase of friction amplitude $\mu$ leads to higher peaks of stationary PDFs. 


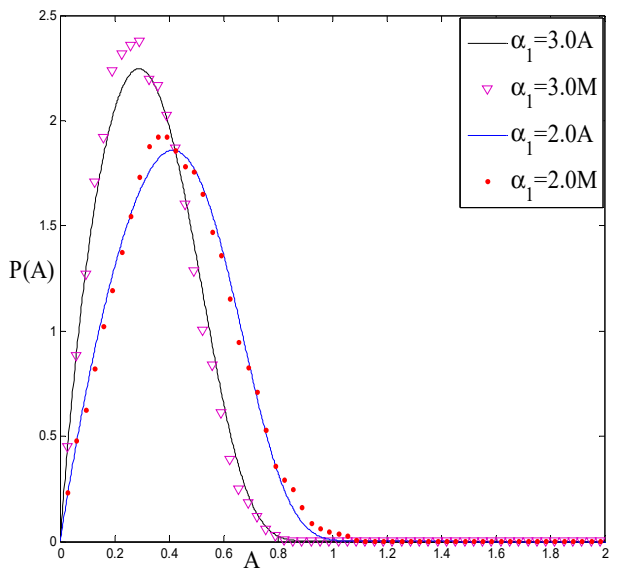

(a)

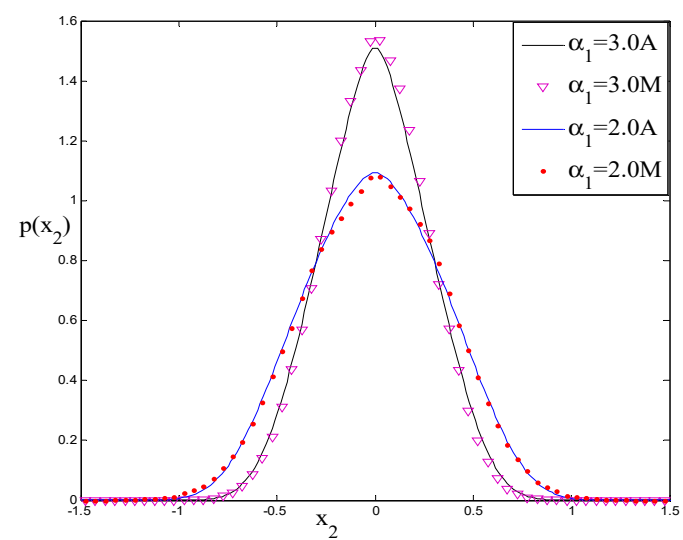

(c)

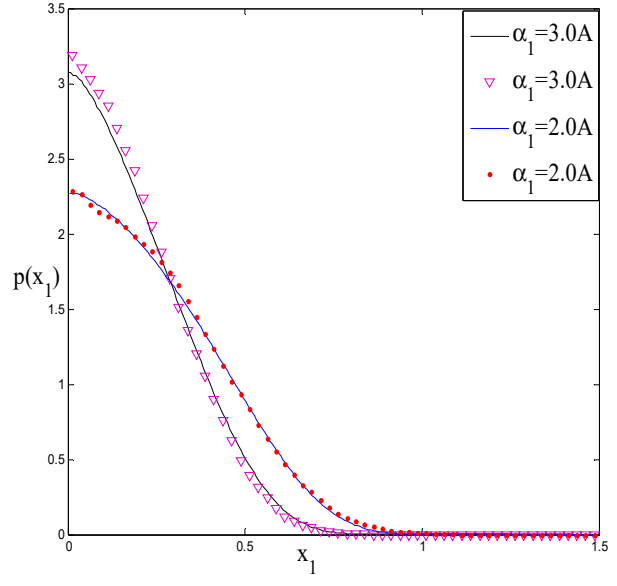

(b)

Figure 3. Numerical and analytical stationary PDF for $D=0.01, \mu=0.001$. (a) PDF of amplitude $A$. (b) PDF of displacement $x_{1}$. (c) PDF of velocity $x_{2}$.

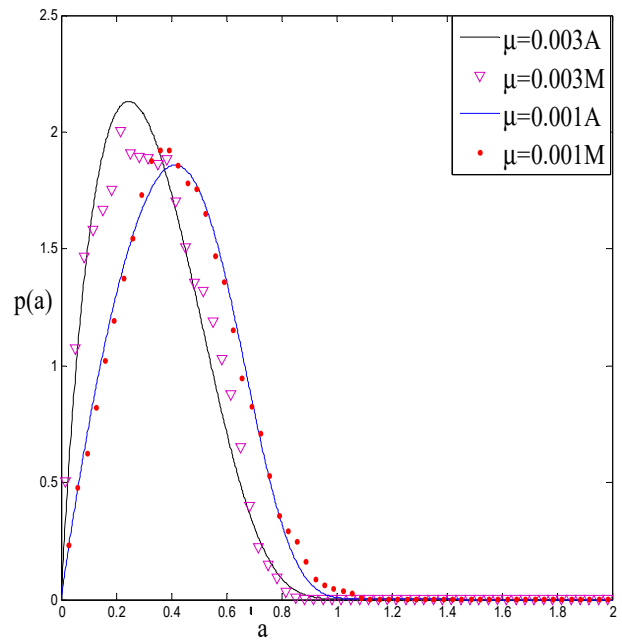

(a)

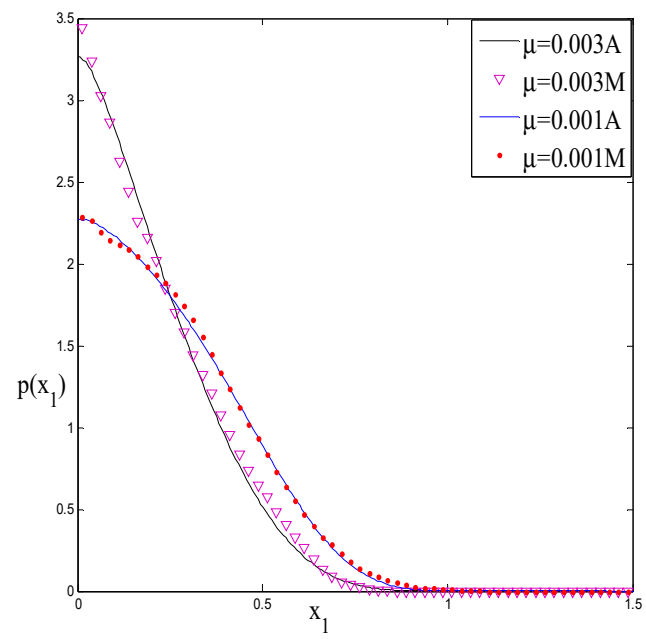

(b)

Figure 4. Cont. 


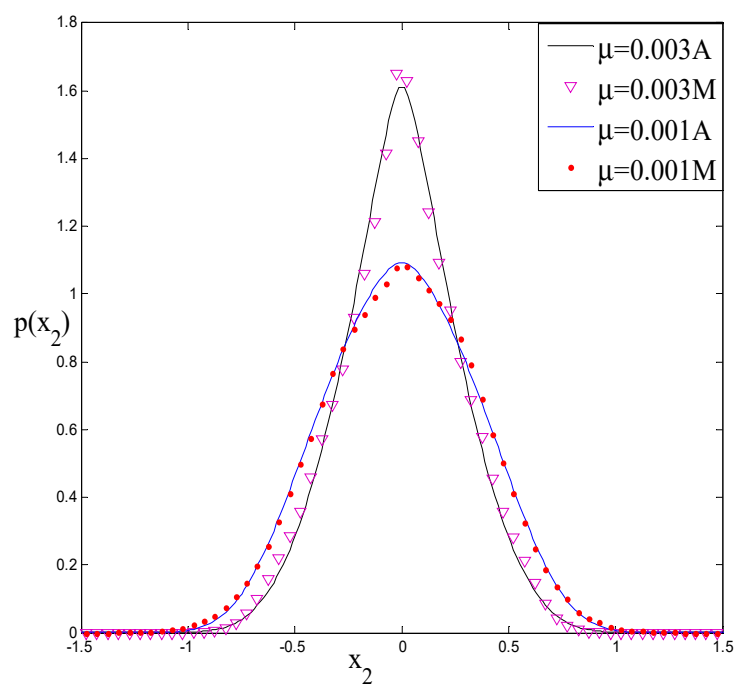

(c)

Figure 4. Numerical and analytical stationary PDF for $\alpha_{1}=2.0, D=0.01$. (a) PDF of amplitude $A$. (b) PDF of displacement $x_{1}$. (c) PDF of velocity $x_{2}$.

By virtue of these figures the accuracy of the analytical method is corroborated by comparison with numerical results. As indicated, the bandwidth, friction amplitude, and noise intensities play a very constructive role in the response of the original system (17).

\subsection{Stochastic Bifurcations}

In this section, when the parameters $c_{2}=0.05, c_{1}=0.025, \alpha=0.6, D=0.01, \omega_{0}=1.0, \alpha_{1}=2.0$ are fixed, friction amplitude $\mu$ and restitution coefficient $r$ are adopted to analyze stochastic P-bifurcation.

Figure 5 shows the influence of the friction amplitude $\mu$ on the stochastic system when the restitution coefficient $r=0.975$ is fixed. The analytical results of joint PDFs on the displacement $x_{1}$ and velocity $x_{2}$ under different friction parameters $\mu$ are exhibited in sub-captions (a-c) of Figure 5. As shown in Figure 5a, there is a singular peak in the equilibrium position for the case of $\mu=0.008$. While $\mu$ is decreased to $\mu=0.001$, one observes the shape of the crater in Figure 5c. This phenomenon demonstrates that stochastic P-bifurcation occurred according to the concept of stochastic bifurcation [30]. Figure $5 \mathrm{~d}$ exhibited the section graphs of analytical results of joint PDFs on the surface $x_{1}=0$ for different values $\mu$. We can conclude that joint PDFs exhibit a transformation from one peak to three peaks when the friction amplitude decreases slowly. Stochastic P-bifurcation occurs as the friction amplitude $\mu$ is close to $\mu \approx 0.038$.

As indicated, Figure $6 \mathrm{a}, \mathrm{b}$ exhibited analytical results of joint PDFs of the displacement $x_{1}$ and velocity $x_{2}$ under different restitution coefficient $r$. The transformation of the joint PDFs from a singular peak to the shape of crater in the equilibrium position indicated stochastic P-bifurcation occurred as the restitution coefficient $r$ varied from $r=0.96$ to $r=0.975$.

Figure $6 c$ exhibited the section graphs of analytical results of joint PDFs on the surface $x_{1}=0$ for different restitution coefficients $r$. In Figure $6 c$, the shape of joint PDFs changed from one peak to two peaks when the restitution coefficient $r$ increases gradually, which mean the appearance of stochastic P-bifurcation occurred. P-bifurcation occurs as the restitution coefficient $r$ is close to $r \approx 0.968$.

Based on the above analysis, it is obvious that the change of friction amplitude $\mu$ and restitution coefficient $r$ can arouse the occurrence of stochastic P-bifurcation. 


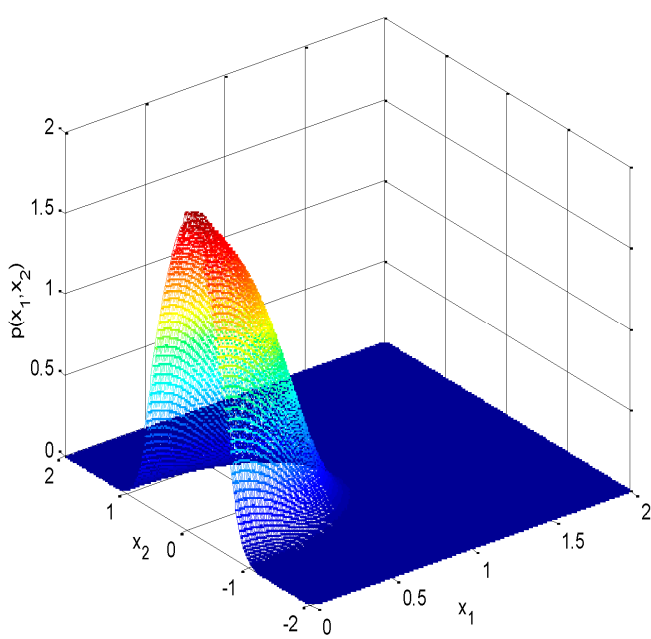

(a)

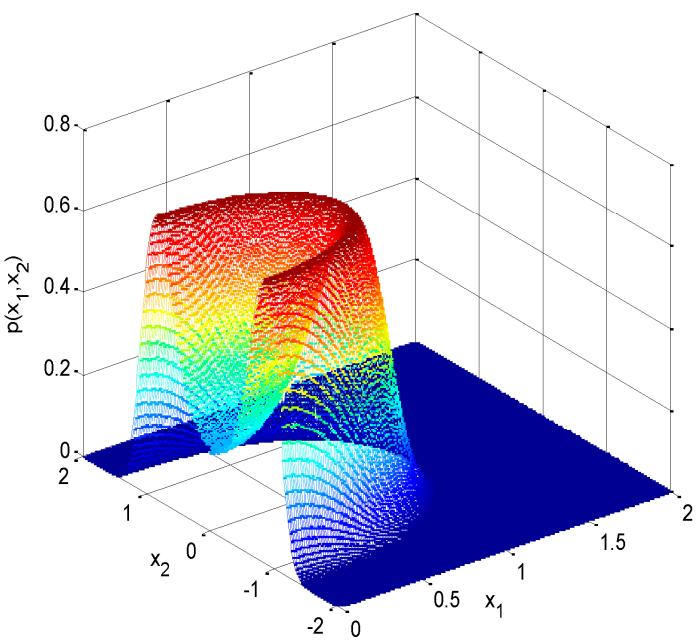

(c)

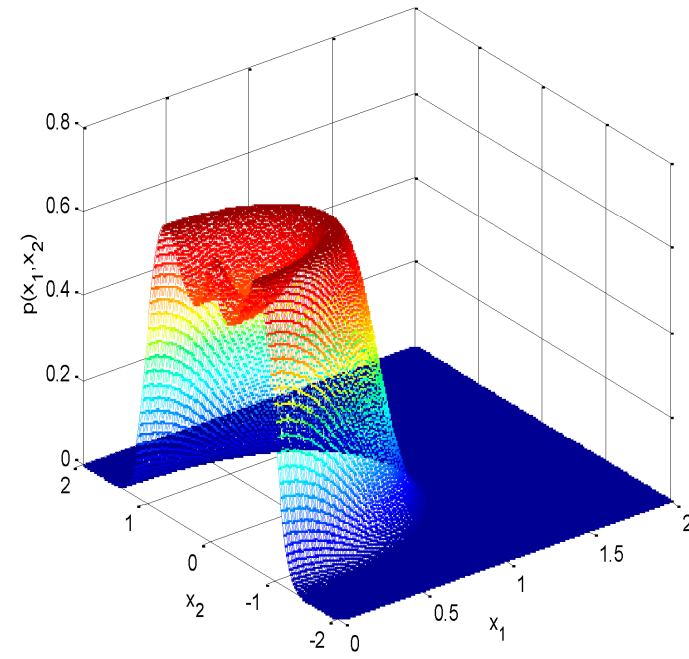

(b)

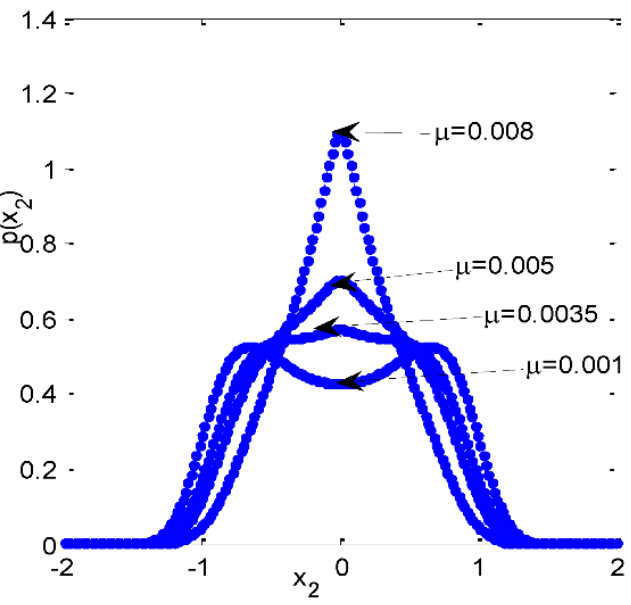

(d)

Figure 5. Joint PDFs of the displacement $x_{1}(t)$ and the velocity $x_{2}(t)$ for $r=0.975$. (a) $\mu=0.08$. (b) $\mu=0.0035$. (c) $\mu=0.001$. (d) Section graphs of joint PDFs on the surface $x_{1}=0$ for different values $\mu$.

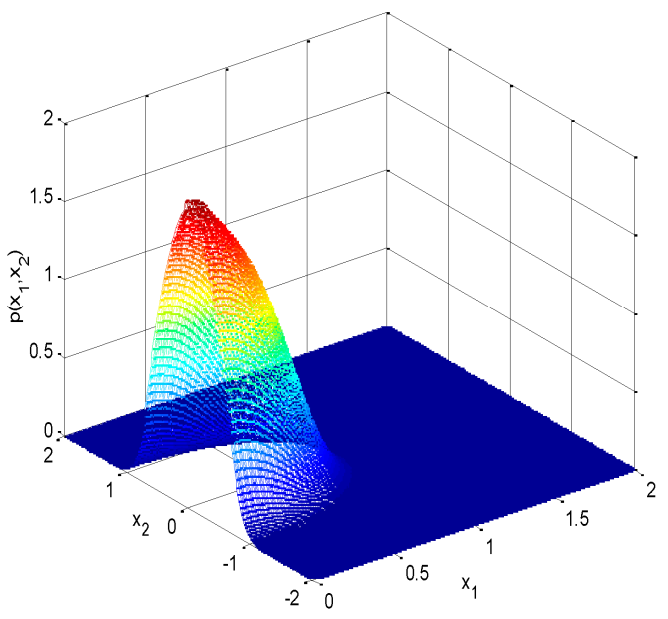

(a)

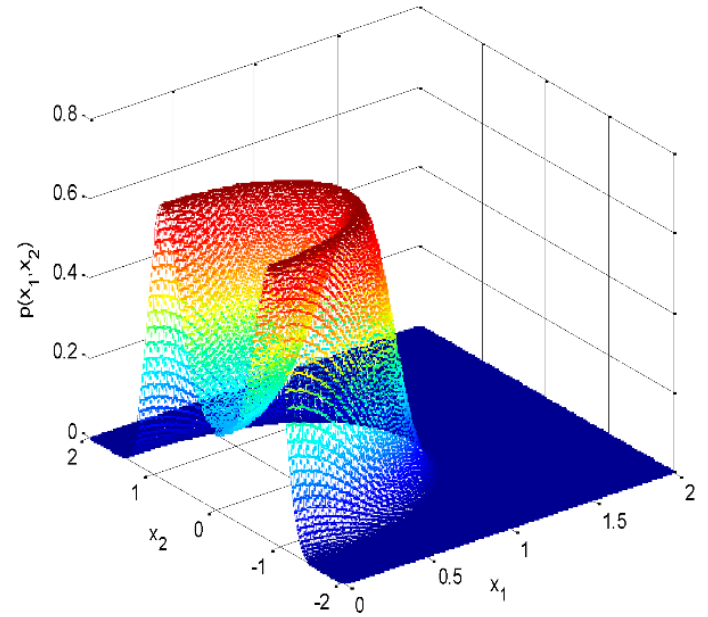

(b)

Figure 6. Cont. 


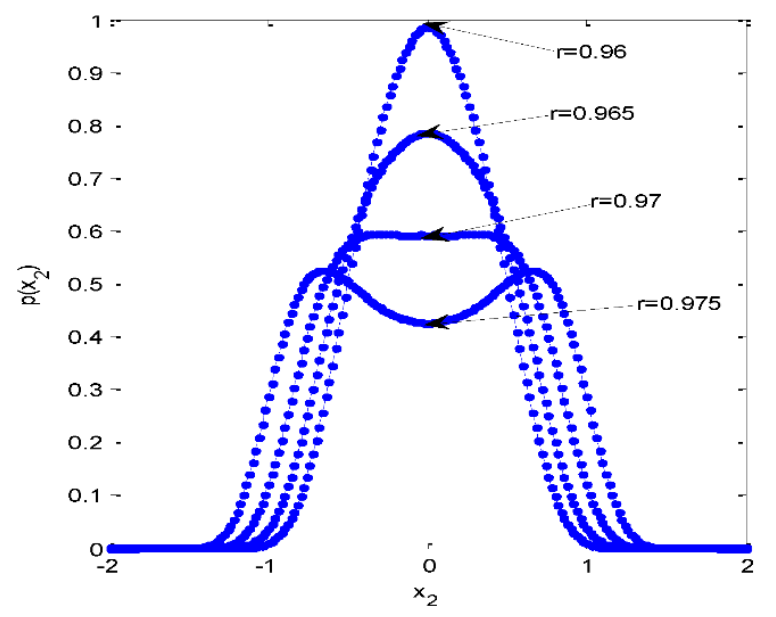

(c)

Figure 6. Joint PDFs of the displacement $x_{1}(t)$ and the velocity $x_{2}(t)$ for $\mu=0.001$. (a) $r=0.96$. (b) $r=0.975$. (c) Section graphs of joint PDFs on the surface $x_{1}=0$ for different values $r$.

\section{Conclusions}

This paper is devoted to researching the stationary response and bifurcation of a non-linear VI system with Coulomb friction excited by real noise. By virtue of the non-smooth transformation, the VI system is converted into a simplified system without barriers. Furthermore, the analytical solutions are derived by the stochastic averaging method. Effects of parameters such as excitation intensity, bandwidth, and friction amplitude are further analyzed in detail on the PDF distribution of the VI system. The analytical results obtained by proposed methods are verified by comparing with the simulated result of original systems. The stochastic P-bifurcation is explored by analyzing the stationary response. The joint PDFs indicate that friction amplitude $\mu$ and restitution coefficient $r$ can arouse the emergence of stochastic P-bifurcation.

Author Contributions: L.L. and W.X. collectively realized theoretical analyses. X.Y. and D.H. computed the numerical simulation results. L.L. wrote the paper. All authors have read and approved the final manuscript.

Funding: This work was supported by the National Natural Science Foundation of China (Grant Nos. 11472212 and 11302170).

Acknowledgments: The authors are very grateful for the comments of the reviewers which helped to improve the present manuscript.

Conflicts of Interest: The authors declare no conflict of interest.

\section{References}

1. Ibrahim, R.A. Modeling Mapping and Application. In Vibro-impact Dynamics; Springer: Berlin, Germany, 2009.

2. Di Bernardo, M.; Nordmark, A.; Olivar, G. Discontinity-induced bifurcations of quilibria in piecewise smooth and impacting dynamical systems. Phys. D Nonlinear Phenom. 2008, 237, 119-136. [CrossRef]

3. Luo, G.W.; Chu, Y.D.; Zhang, Y.L.; Zhang, J.G. Double Neimark-Sacker bifurcation and torus bifurcation of a class of vibratory systems with symmetrical rigid stops. J. Sound Vib. 2006, 298, 154-179. [CrossRef]

4. Wagg, D.J.; Bishop, S.R. Chatter sticking and chaotic impacting motion in a two degree of freedom impact oscillator. Int. J. Bifurc. Chaos 2001, 11, 57-71. [CrossRef]

5. Namachchivaya, N.S.; Park, J.H. Stochastic dynamics of impact oscillators. J. Appl. Mech. 2005, 72, 862-870. [CrossRef]

6. Park, J.H.; Namachchivaya, N.S. Noisy impact oscillators. In Proceedings of the ASME 2004 International Mechanical Engineering Congress and Exposition, Anaheim, CA, USA, 13-19 November 2004.

7. Huang, Z.; Liu, Z.; Zhu, W. Stationary response of multi-degree-of-freedom vibro-impact systems under white noise excitation. J. Sound Vib. 2004, 275, 223-240. [CrossRef] 
8. Xu, M.; Wang, Y.; Jin, X.; Huang, Z.; Yu, T. Random response of vibro-impact systems with inelastic contact. Int. J. Non-Linear Mech. 2013, 52, 26-31. [CrossRef]

9. Rong, H.; Wang, X.; Xu, W.; Fang, T. Subharmonic response of a single-degree-of freedom nonlinear 8vibro-impact system to a randomly disordered periodic excitation. J. Sound Vib. 2009, 327, 173-182. [CrossRef]

10. Yang, G.; Xu, W.; Jia, W.; He, M. Random vibrations of Rayleigh vibroimpact oscillator under parametric poisson white noise. Commun. Nonlinear Sci. Numer. Simul. 2016, 33, 19-29. [CrossRef]

11. Zhu, H. Stochastic response of vibro-impact Duffing oscillators under external and parametric Gaussian white noises. J. Sound Vib. 2013, 333, 945-961. [CrossRef]

12. Zhu, H. Probabilistic solution of vibro-impact stochastic Duffing systems with a unilateral non-zero offset barrier. Phys. A Stat. Mech. Appl. 2014, 40, 335-344. [CrossRef]

13. Green, P.L.; Worden, K.; Sims, N.D. On the identification and modeling of friction in a randomly excited energy harvester. J. Sound Vib. 2013, 332, 4696-4708. [CrossRef]

14. Sun, J.Q. Random vibration analysis of a non-linear system with dry friction damping by the short-time Gaussian cell mapping method. J Sound Vib. 1995, 180, 785-795. [CrossRef]

15. Kumar, P.; Narayanan, S.; Gupta, S. Stochastic bifurcation analysis of a Duffing oscillator with Coulomb friction excited by Poisson White noise. Procedia Eng. 2016, 144, 998-1006. [CrossRef]

16. Sun, J.J.; Xu, W.; Lin, Z.F. Research on the reliability of friction system under combined additive and multiplicative random excitations. Commun. Nonlinear Sci. Numer. Simul. 2018, 54, 1-12. [CrossRef]

17. Rigatos, G.G.; Siano, P. Sensorless control of electric motors with Kalman Filters: Applications to robotic and industrial system. Int. J. Adv. Robot. Syst. 2011, 8, 71. [CrossRef]

18. Rigatos, G.; Siano, P. Sensorless nonlinear control of induction motors using Unscented Kalman Filtering. In Proceedings of the IECON 2012-38th Annual Conference on IEEE Industrial Electronics Society, Montreal, QC, Canada, 25-28 October 2012; pp. 4654-4659.

19. Bryson, A.E.; Ho, Y.-C. Applied Optimal Control: Optimization, Estimation and Control. Routledge: London, UK, 1935.

20. Pappalardo, C.M.; Guida, D. Use of the Adjoint Method for Controlling the Mechanical Vibrations of Nonlinear Systems. Machines 2018, 6, 19. [CrossRef]

21. Pappalardo, C.M.; Guida, D. System algorithm for computing the Modal Parameters of linear mechanical Systems. Machines 2018, 6. [CrossRef]

22. Ibrahim, R.A. Vibro-Impact Dynamics Modeling, Mapping and Applications; Springer: Berlin, Germany, 2009.

23. Dimentberg, M.F.; Iourtchenko, D.V. Random vibrations with impacts: A review. Nonlinear Dyn. 2004, 36, 229-254. [CrossRef]

24. Zhu, W.Q.; Huang, Z.L.; Suzuki, Y. Response and stability of strongly non-linear oscillators under wide-band random excitation. Int. J. Non-Linear Mech. 2011, 36, 1235-1250. [CrossRef]

25. Zhu, W.; Cai, G. Random vibration of viscoelastic system under broad-band excitations. Int. J. Non-Linear Mech. 2011, 46, 720-726. [CrossRef]

26. Ling, Q.; Jin, X.; Huang, Z. Response and stability of SDOF viscoelastic system under wideband noise excitations. J. Franklin Inst. 2008, 345, 499-507. [CrossRef]

27. Zhuravlev, V.F. A method for analyzing vibration-impact systems by means of special function. Mech. Solids 1976, 11, 23-27.

28. Stratonovich, R.L. Topics in the Theory of Random Noise; Gordon Breach: New York, NY, USA, 1963.

29. Khasminskii, R.Z. A limit theorem for the solution of differential equations with random right-band sides. Theory Probab. Appl. 1966, 11, 390-405. [CrossRef]

30. Xu, W.; He, Q.; Rong, H.; Fang, T. Global analysis of stochastic bifurcation in Ueda system. In Proceedings of the Fifth International Conference on stochastic Structural Dynamics-SSD03, Hangzhou, China, 26-28 May 2003; pp. 509-515.

(C) 2018 by the authors. Licensee MDPI, Basel, Switzerland. This article is an open access article distributed under the terms and conditions of the Creative Commons Attribution (CC BY) license (http://creativecommons.org/licenses/by/4.0/). 